\title{
Natural Language Stage of Change Modelling for "Motivationally-driven" Weight Loss Support
}

\author{
Selina Meyer \\ selina.meyer@ur.de \\ Chair for Information Science, University of Regensburg \\ Regensburg, Bavaria, Germany
}

\begin{abstract}
Motivational factors play a significant role in weight loss. Providing appropriate support based on an individual's motivational level could be a deciding factor in weight loss success. Considering the high cost of obesity to public health care, a system with the ability to assess motivation could benefit individuals and the public alike. As self-report measures are not always available and would be tedious to use on a regular basis, one way to achieve this understanding could be the interpretation of natural language. This project studies the feasibility of using conversational agents to measure motivation by engaging users in natural conversation during weight loss. This approach could be a good means of tracking motivation, as it is cheap and accessible and can model motivation in a more natural and engaging way than self-report measures.
\end{abstract}

\section{CCS CONCEPTS}

- Human-centered computing $\rightarrow$ Natural language interfaces; - Computing methodologies $\rightarrow$ Information extraction; $\bullet$ Applied computing $\rightarrow$ Sociology.

\section{KEYWORDS}

Conversational AI, Empathetic Dialogue, Weight Loss, Motivation

\section{ACM Reference Format:}

Selina Meyer. 2021. Natural Language Stage of Change Modelling for "Motivationally-driven" Weight Loss Support. In Proceedings of the 2021 International Conference on Multimodal Interaction (ICMI '21), October 18-22, 2021, Montréal, QC, Canada. ACM, New York, NY, USA, 5 pages. https: //doi.org/10.1145/3462244.3481279

\section{INTRODUCTION}

In recent decades, overweight and obesity have become a growing societal problem. According to the World Health Organization's most recent estimates, 39\% of the world's population are overweight and $13 \%$ are obese [36]. At the current rate, we can expect the global obesity rate to rise to $20 \%$ by 2025 [37]. Weight problems can decrease quality of life and put individuals at increased risk for various other diseases such as coronary heart disease, type 2 diabetes and cancer and increase overall mortality [5]. As a result,

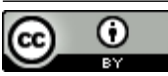

This work is licensed under a Creative Commons Attribution International 4.0 License.

ICMI '21, October 18-22, 2021, Montréal, QC, Canada

(c) 2021 Copyright held by the owner/author(s).

ACM ISBN 978-1-4503-8481-0/21/10.

https://doi.org/10.1145/3462244.3481279 more than $13 \%$ of global total healthcare expenditure is attributable to high BMI [37].

While a wide variety of treatment options and weight loss programmes exist [13, 15, 33], most non-surgical treatments tend to suffer from a high dropout rate, resulting in relatively low success rates [2, 16, 20]. Even though factors that influence weight and weight loss are manifold and cannot be reduced to a single predictor $[5,10]$, we know that motivational factors, such as a person's readiness to change and self-efficacy, are crucial elements to weight loss success [21, 31, 32, 35] . Motivational intervention is showing promise in weight loss treatment [29] and counselling approaches such as Motivational Interviewing (MI) have been used in the context of physical activity and nutrition for some time [6].

Depending on a person's level of motivation and stage of change, different treatment methods are best suited to support behavioural changes [1]. For instance, to encourage people with lower motivation levels and high degrees of ambivalence, the first priority should be to resolve this ambivalence, while individuals with higher levels of motivation and in later stages of change will benefit more from developing specific skills and planning further steps in their behaviour change [1, 6, 23]. However, motivation and related concepts undergo frequent fluctuations during prolonged behaviour change as it is required in weight loss, which can often result in relapses or retrograde steps [3, 27, 32]. In clinical studies exploring the relationship between motivation and weight loss success, motivation is usually measured using one or several multi-item self-report questionnaires, i.e. $[8,29,34]$. This approach is not feasible in practice, however, as behaviour change is inherently difficult [3] and additional workload should arguably be kept to a minimum. Expecting clients to fill out long questionnaires on a regular basis may thus in itself become an inhibitor to motivation and sustainable behaviour change.

Traditional counselling approaches such as MI successfully rely on natural conversation as a means to recognize motivation and readiness to change $[6,23]$. Thus, natural language together with the framework provided by such a counselling approach could offer valuable clues to an individual's level of motivation. Consequently, a conversational agent (CA) that maintains dialogue with individuals during weight loss may be a good means of tracking motivational levels in a naturalistic and engaging way, hence eliciting useful information that can enable the CA to provide appropriate support and interventions.

In order to build such a system, it is first necessary to learn how exactly motivational levels and stages of change can be mapped to natural written conversation. To achieve this, different features of natural language may be explored as predictor variables for motivation. It will also be important to explore differences between 
human-human conversation and human-chatbot conversation in this context. While research shows, that self-disclosure to a chatbot has the same effects as to a person [18], we also know that human-chatbot communication is typically expressed in a different way than human-human communication [17]. Consequently, this project is driven by the following research questions:

$R Q 1:$ How is weight loss motivation voiced in written conversation?

RQ2: How much and what context is necessary to understand individuals' motivational levels during weight loss, or more specifically:

a. What features help predict a person's current motivation for and thoughts about weight loss?

b. Can a person's motivational level and stage of change be predicted by their utterances?

RQ3: How can fluctuation in motivation over time be modeled, measured and recognized?

$R Q 4:$ In what ways does written articulation of motivation change in human-chatbot interaction?

\section{BACKGROUND AND RELATED WORK}

Most technology-delivered weight loss support systems focus on meal and activity tracking, information initiatives or gamification strategies [4, 19, 24, 26]. However, such systems do not aim at understanding the user or the emotional and motivational processes involved in weight loss. As such, they are unable to adapt to a user's individual needs and deal with common fluctuation in motivation. They mainly aim at facilitating weight loss as long as the user is "on-track" and largely disregard the problem of attrition common in most weight loss programmes.

In contrast, MI heavily relies on understanding and supporting individuals in the process of change [23]. Originally developed for addiction treatment, it has since been successfully applied to a large number of contexts, including weight loss therapy [6]. Its goal is to help the client to identify individual reasons for change by resolving ambivalence and thus increasing motivation and readiness to change [6,23]. Ambivalence can be recognized by looking out for two opposing signals that help to contextualize the client's motivations and give experienced counsellors clues about the client's stage of change. The first signal, "change talk", includes all utterances, that are positively associated with the change, e.g. reasons why it is necessary, recognition of strengths that might help, trust in ability to change, concrete strategies, plans and successes, etc. As such, change talk can be seen to be a positive marker for a person's willingness to change and should be actively elicited by the qualified counsellor [6, 23]. In opposition to change talk, users may also express "sustain talk", meaning comments "that support the status quo" [6, p. 15]. This kind of talk encompasses doubts and fears associated with the change, expectations of failure, reasons or excuses why change cannot be pursued, references to past failures and attempts to play down the problem, among other things (see table 1 for examples). When ambivalence is strong, change and sustain talk often occur closely together, symbolizing the client's conflict of emotions about the impending behaviour change [23] The transtheoretical model ties in with the concept of MI and further conceptualizes the process of behaviour change. It suggests that people undergo different stages of change, starting with precontemplation, where a person is not aware of the necessity to change their behaviour, over contemplation, where first intents of behaviour change form and ending in maintenance and termination, where changed behaviour has been stable for a certain amount of time [27]. While the original model encompasses 6 stages of change, in the literature and corresponding questionnaires, it is sometimes reduced to 3 - 5 stages depending on the context, with precontemplation, contemplation and action as essential phases which are always present $[9,14,23]$. Being able to recognize the opposing patterns of change and sustain talk in a conversation around change and contextualize them within the transtheoretical model could be a first step towards modelling motivation.

Table 1: Examples of change talk, sustain talk and ambivalence in naturalistic written conversation data (Translated from German by the author. Data available on request.)

\begin{tabular}{l|l} 
& Example \\
\hline \hline change talk & $\begin{array}{l}\text { "I'm worth feeling this way and I'll be damned if } \\
\text { I'll ever forget it. I'm worth it." (utterance 291/10) } \\
\text { "this shapeless thick body, I just find it disgusting." } \\
\text { (utterance 671/5) } \\
\text { "Starting tomorrow, I'll be cooking for myself } \\
\text { again, so I can plan for more vegetables." (ut- } \\
\text { terance 722/14) }\end{array}$ \\
\hline sustain talk & $\begin{array}{l}\text { "I just can't manage to cook healthily with the } \\
\text { money I have" (utterance 261/4) } \\
\text { "the month is not even over, but I have already } \\
\text { written it off" (utterance 869/8) }\end{array}$ \\
\hline ambivalence & $\begin{array}{l}\text { "I am a regular carbohydrate junkie. I do not want } \\
\text { it myself, but just can't get it together." (utter- } \\
\text { ances 333/3-4) }\end{array}$
\end{tabular}

There has been research on the effects of MI administered by CAs, which generally showed positive impacts on participants $[7,11,25,28]$. However, so far this approach has largely been used to guide CAs actions. We believe, that in order to successfully deliver automated MI and helping people in their behaviour changes and weight loss journeys, it is important to gain an in-depth understanding of the processes involved in behaviour change and how different states of mind and motivational levels are communicated by the individuals undergoing the change. To achieve this, we plan to employ concepts from $\mathrm{MI}$ in such a way, that we can reach a true understanding of a user's motivational level and current stage of change through their interaction with a CA. To the author's knowledge there is currently very little research focusing on recognizing and interpreting motivational levels via natural language processing. In fact, we could only find very little research on the automatic modelling of motivation in general, regardless of context and used predictors $[12,22]$. In a study exploring natural language processing methods to code MI, classification of change talk and sustain talk was unreliable, with kappa agreement between algorithms and humans ranging only between 0.2 and 0.3 [30]. Improving on these results and finding a way to reliably code user utterances automatically would be a first step towards understanding the user 
and thus towards being able to offer emotional support tailored to the user in a given situation.

\section{RECOGNIZING MOTIVATION AND STAGES OF CHANGE}

We hypothesize that, given enough information, it is possible to automatically deduct a person's level of motivation and stage of change by interpreting their utterances, for example by the presence and absence of change and sustain talk. As a first step towards recognizing such utterances, we crawled naturalistic written conversation data from Germany's largest weight loss forum "adipositas24.de" ${ }^{1}$. The posts were written between May $1^{\text {st }}, 2006$ and July $31^{\text {st }} 2020$ and sourced from two sub-forums related to weight loss without surgery. Detailed examination of the posts revealed two main purposes of posting in the forum:

P1. To share and receive practical and factual information and strategies

P2. To seek or provide emotional support, and share motivation, success and setbacks

We first identified posts, which were related to motivation, meaning that they contained at least one instance of change talk or sustain talk. Only $17 \%$ of posts (1.222) were found to relate to motivation. The other posts were largely part of P1. In some cases, they had nothing to do with weight loss at all and were thus deemed as irrelevant for this research.

In order to address $R Q 1$, the remaining "motivation posts" will be annotated on a sentence to sentence basis using four first-level categories: 1. Change Talk, 2. Sustain Talk, 3. Nothing, 4. Both/Ambivalence.

\subsection{Change talk recognition and scaling}

Once enough posts have been annotated, we plan on applying machine learning algorithms as a means to recognize change and sustain talk in written conversation. In this, we aim to improve on [30]'s approaches and results, by applying up-to-date transformer models, which are likely to yield better results than the RNN and DSF tested in the cited work. Additionally, by solely focusing on client codes (change and sustain talk) and leaving out counsellor codes, which were also subject of annotation in [30], we limit the algorithm's scope and the number of labels, which is likely to increase prediction accuracy. The classifier will also be tested on content from other sub-forums, to ensure a certain degree of context independence. We are also considering semi-supervised learning as a way to achieve scalability of our dataset. Having a larger dataset for reference will help with user utterance classification in later stages of the project and also serve as a control sample that identifies the system's ability to correctly classify utterances outside of our small initial dataset.

\subsection{Mapping utterances to motivational levels}

To explore if and how a person's stage of change can be estimated by their utterances, we are planning a crowd-sourcing study in which overweight and obese participants will be required to complete a questionnaire measuring their stage of change [14], after which they will be presented with sentences of the annotated forum data

\footnotetext{
${ }^{1}$ https://www.adipositas24.de
}

and asked to what extend they agree with the statements and how likely it is that they would write something similar in their specific situation. This step will help to address $R Q 2$ as we will gain insight on what kind of utterances are likely to be voiced in the different stages of change.

\subsection{Modelling fluctuations in motivation and providing "motivationally-driven" support}

Results from the study could help us estimate a person's stage of change by interpreting their utterances. The information gained can be put to use by creating a rule-based chatbot that has two main purposes:

- Measure motivation and its fluctuations over time

Each user utterance could be allocated a motivation-score. When calculating this score, we could take into account how likely it is that a person in a certain stage of change voices such an utterance, as indicated by the results of the study outlined in 3.2 as well as how the utterance is classified. As a simple example if the user writes "this shapeless thick body, I just find it disgusting." (see table 1), the system would first check, for which stage this is a typical sentence and then for instance attribute it to the contemplation phase, thus giving it 0 points (see table 2). Next, it would classify the statement as change talk and add 2 points, to get a motivation score of 2 . The weighted mean of all user-utterances, where more current

Table 2: Example motivation score calculation

\begin{tabular}{lll} 
likely stage & + & type of utterance \\
\hline precontemplation $(-1)$ & sustain talk $(-1)$ \\
contemplation $(0)$ & nothing $(0)$ \\
action $(+1)$ & both $(+1)$ \\
& change talk $(+2)$
\end{tabular}

utterances are weighted stronger than less recent ones, can then be used to estimate the user's stage of change or motivational level. How many points to allocate to the different stages and utterance types will be defined later on. We will have to consider whether the stage and type of utterance should be weighted differently and how fine-grained the motivation score should be. This highly depends on the results of the crowd-sourcing study. Once functional, this approach could yield information about motivational fluctuations over time and, if tested on enough users over some time, might allow us to identify different "change-types" and thus help to answer RQ3.

- Provide motivationally-driven weight loss support

Based on the estimated stage of change, and how motivation has been developing, the chatbot could then employ different support strategies (see table 3). This requires the motivational level to constantly be re-evaluated by the system.

\section{CONCLUSION}

This abstract outlines doctoral research in its beginning stages. We described a tentative research plan and the initial data collection which mainly serves as preparation for the remaining project. Our goal is to gain a deeper understanding of motivation and use this understanding to provide motivationally-driven support in the specific context of weight loss. To achieve this, we are planning to apply 
Table 3: Strategies to use in different motivational stages

\begin{tabular}{l|l} 
Stage & Strategy \\
\hline \hline low motivation/ \\
precontemplation & $\begin{array}{l}\text { Raise awareness } \\
\text { Get Person to talk about prob- } \\
\text { lems their weight causes them }\end{array}$ \\
\hline increasing motivation/ & $\begin{array}{l}\text { Reinforce change talk } \\
\text { Give examples of benefits losing } \\
\text { weight could have }\end{array}$ \\
\hline high motivation/ & $\begin{array}{l}\text { Set goals } \\
\text { Track achievements } \\
\text { action }\end{array}$ \\
Plan weight loss strategies \\
\hline decreasing motivation/ \\
relapse & $\begin{array}{l}\text { Raise awareness } \\
\text { Remind of past achievements } \\
\text { and current successes } \\
\text { Reinforce change talk }\end{array}$ \\
&
\end{tabular}

concepts from established counselling methods to natural language processing. We hope that this new approach can add to the existing research and improve the efficiency and capacity for empathy of automated weight loss support by adding a deeper understanding of motivation and its underlying processes and fluctuations.

\section{REFERENCES}

[1] Vickie Z Beckwith and Jennifer Beckwith. 2020. Motivational Interviewing: A Communication Tool to Promote Positive Behavior Change and Optimal Health Outcomes. NASN School Nurse 35, 6 (2020), 344-351.

[2] S. C. Bischoff, A. Damms-Machado, C. Betz, S. Herpertz, T. Legenbauer, T. Löw, J. G. Wechsler, G. Bischoff, A. Austel, and T. Ellrott. 2012. Multicenter evaluation of an interdisciplinary 52-week weight loss program for obesity with regard to body weight, comorbidities and quality of life - A prospective study. Int. 7 . Obes. 36, 4 (2012), 614-624. https://doi.org/10.1038/ijo.2011.107

[3] Mark E Bouton. 2014. Why behavior change is difficult to sustain. Preventive medicine 68 (2014), 29-36.

[4] Jacky Casas, Elena Mugellini, and Omar Abou Khaled. 2018. Food diary coaching chatbot. In Proceedings of the 2018 ACM International foint Conference and 2018 International Symposium on Pervasive and Ubiquitous Computing and Wearable Computers. 1676-1680.

[5] Centers for Disease Control and Prevention. 2021. Adult Obesity Causes \& Consequences. https://www.cdc.gov/obesity/adult/causes.html

[6] Dawn Clifford and Laura Curtis. 2016. Motivational interviewing in nutrition and fitness. Guilford Publications.

[7] Joana Galvão Gomes da Silva, David J Kavanagh, Tony Belpaeme, Lloyd Taylor, Konna Beeson, and Jackie Andrade. 2018. Experiences of a motivational interview delivered by a robot: qualitative study. Fournal of medical Internet research 20, 5 (2018), e116.

[8] John B Dixon, Cheryl P Laurie, Margaret L Anderson, Melissa J Hayden, Maureen E Dixon, and Paul E O'brien. 2009. Motivation, readiness to change, and weight loss following adjustable gastric band surgery. Obesity 17, 4 (2009), 698705 .

[9] David JA Dozois, Henny A Westra, Kerry A Collins, Tak S Fung, and Jennifer KF Garry. 2004. Stages of change in anxiety: Psychometric properties of the University of Rhode Island Change Assessment (URICA) scale. Behaviour research and therapy 42,6 (2004), 711-729.

[10] Kristina Elfhag and Stephan Rössner. 2005. Who succeeds in maintaining weight loss? A conceptual review of factors associated with weight loss maintenance and weight regain. Obesity reviews 6, 1 (2005), 67-85.

[11] Stijn Friederichs, Catherine Bolman, Anke Oenema, Janneke Guyaux, and Lilian Lechner. 2014. Motivational interviewing in a web-based physical activity intervention with an avatar: randomized controlled trial. fournal of medical Internet research 16, 2 (2014), e48.

[12] Yoshimi Fukuoka, Teri G Lindgren, Yonatan Dov Mintz, Julie Hooper, and Anil Aswani. 2018. Applying natural language processing to understand motivational profiles for maintaining physical activity after a mobile app and accelerometerbased intervention: the MPED randomized controlled trial. FMIR mHealth and uHealth 6, 6 (2018), e10042.

[13] Kimberly A Gudzune, Ruchi S Doshi, Ambereen K Mehta, Zoobia W Chaudhry, David K Jacobs, Rachit M Vakil, Clare J Lee, Sara N Bleich, and Jeanne M Clark.
2015. Efficacy of commercial weight-loss programs: an updated systematic review. Annals of internal medicine 162, 7 (2015), 501-512.

[14] Gregor Hasler, Richard Klaghofer, and Claus Buddeberg. 2003. Der Fragebogen zur Erfassung der Veränderungsbereitschaft (FEVER). PPmP-Psychotherapie. Psychosomatik - Medizinische Psychologie 53, 9/10 (2003), 406-411.

[15] H. Hauner, A. Moss, A. Berg, S. C. Bischoff, M. Colombo-Benkmann, T. Ellrott, C. Heintze, U. Kanthak, D. Kunze, N. Stefan, M. Teufel, M. Wabitsch, and A. Wirth. 2014. Interdisziplinäre Leitlinie der Qualität S3 zur „Prävention und Therapie der Adipositas”. Technical Report 04. Deutsche Adipositas-Gesellschaft (DAG) e.V., Martinsried. 60-63 pages. https://doi.org/10.1055/s-0037-1618857

[16] Erik Hemmingsson, Kari Johansson, Jonas Eriksson, Johan Sundström, Martin Neovius, and Claude Marcus. 2012. Weight loss and dropout during a commercial weight-loss program including a very-low-calorie diet, a low-calorie diet, or restricted normal food: observational cohort study. The American journal of clinical nutrition 96, 5 (2012), 953-961.

[17] Jennifer Hill, W Randolph Ford, and Ingrid G Farreras. 2015. Real conversations with artificial intelligence: A comparison between human-human online conversations and human-chatbot conversations. Computers in human behavior 49 (2015), 245-250.

[18] Annabell Ho, Jeff Hancock, and Adam S Miner. 2018. Psychological, relational, and emotional effects of self-disclosure after conversations with a chatbot. fournal of Communication 68, 4 (2018), 712-733.

[19] Samuel Holmes, Anne Moorhead, Raymond Bond, Huiru Zheng, Vivien Coates, and Mike McTear. 2018. WeightMentor: a new automated chatbot for weight loss maintenance. In Proceedings of the 32nd International BCS Human Computer Interaction Conference 32.1-5.

[20] Jeffery J Honas, James L Early, Doren D Frederickson, and Megan S O'brien. 2003. Predictors of attrition in a large clinic-based weight-loss program. Obesity research 11, 7 (2003), 888-894.

[21] Jutta Mata, Marlene N Silva, Paulo N Vieira, Eliana V Carraça, Ana M Andrade, Sílvia R Coutinho, Luis B Sardinha, and Pedro J Teixeira. 2011. Motivational "spill-over" during weight control: Increased self-determination and exercise intrinsic motivation predict eating self-regulation. Health Psychology 28, 6 (2011), 709-716.

[22] David Melhart, Ahmad Azadvar, Alessandro Canossa, Antonios Liapis, and Georgios N Yannakakis. 2019. Your gameplay says it all: modelling motivation in Tom Clancy's the division. In 2019 IEEE Conference on Games (CoG). IEEE, 1-8.

[23] William R. Miller and Stephen Rollnick. 2002. Motivational interviewing: Preparing people for change. Guilford Press.

[24] Madison Milne-Ives, Ching Lam, Caroline De Cock, Michelle Helena Van Velthoven, and Edward Meinert. 2020. Mobile apps for health behavior change in physical activity, diet, drug and alcohol use, and mental health: Systematic review. FMIR mHealth and uHealth 8, 3 (2020), e17046.

[25] Johanna Nurmi, Keegan Knittle, Todor Ginchev, Fida Khattak, Christopher Helf, Patrick Zwickl, Carmina Castellano-Tejedor, Pilar Lusilla-Palacios, Jose CostaRequena, Niklas Ravaja, et al. 2020. Engaging users in the behavior change process with digitalized motivational interviewing and gamification: development and feasibility testing of the precious app. FMIR mHealth and uHealth 8, 1 (2020), e12884.

[26] Christine A Pellegrini, Jennifer M Duncan, Arlen C Moller, Joanna Buscemi, Alyson Sularz, Andrew DeMott, Alex Pictor, Sherry Pagoto, Juned Siddique, and Bonnie Spring. 2012. A smartphone-supported weight loss program: design of the ENGAGED randomized controlled trial. BMC public health 12, 1 (2012), 1-10.

[27] James O Prochaska and Bess H Marcus. 1994. The transtheoretical model: Applications to exercise. In Advances in exercercise adherence. Human Kinetics Publishers, 161-180.

[28] Daniel Schulman, Timothy W Bickmore, and Candace L Sidner. 2011. An Intelligent Conversational Agent for Promoting Long-Term Health Behavior Change Using Motivational Interviewing. In AAAI Spring Symposium: AI and Health Communication.

[29] Carolina Domingues Segallaa, Raquel de Melo Boffb, Ana Maria Pandolfo Feolia, Andreia da Silva Gustavoa, Márcio Vinícius Fagundes Donadioa, and Margareth da Silva Oliveiraa. 2020. Maintenance of Motivation for Lifestyle Change in Adolescents with Overweight Obesity: A 6-Month Follow-Up Study. Revista Argentina de Clínica Psicológica 29, 3 (2020), 47-57.

[30] Michael Tanana, Kevin A Hallgren, Zac E Imel, David C Atkins, and Vivek Srikumar. 2016. A comparison of natural language processing methods for automated coding of motivational interviewing. Fournal of substance abuse treatment 65 (2016), 43-50.

[31] PJ Teixeira, Scott B Going, LB Sardinha, and TG2005 Lohman. 2005. A review of psychosocial pre-treatment predictors of weight control. obesity reviews 6, 1 (2005), 43-65.

[32] Pedro J Teixeira, Marlene N Silva, Jutta Mata, António L Palmeira, and David Markland. 2012. Motivation, self-determination, and long-term weight control. International fournal of Behavioral Nutrition and Physical Activity 9, 1 (2012), $1-13$.

[33] Adam Gilden Tsai and Thomas A Wadden. 2005. Systematic review: an evaluation of major commercial weight loss programs in the United States. Annals of internal 
medicine 142,1 (2005), 56-66.

[34] Maité Verloigne, Ilse De Bourdeaudhuij, Ann Tanghe, Eva D'Hondt, Lotte Theuwis, Maarten Vansteenkiste, and Benedicte Deforche. 2011. Self-determined motivation towards physical activity in adolescents treated for obesity: an observational study. International fournal of Behavioral Nutrition and Physical Activity 8, 1 (2011), 1-11.

[35] Geoffrey C Williams, Virginia M Grow, Zachary R Freedman, Richard M Ryan, and Edward L Deci. 1996. Motivational predictors of weight loss and weight-loss maintenance. Fournal of personality and social psychology 70, 1 (1996), 115-126.

[36] World Health Organization. 2020. Obesity and Overweight. https://www.who. $\mathrm{int} /$ news-room/fact-sheets/detail/obesity-and-overweight

[37] World Obesity Federation. 2020. Obesity: missing the 2025 global targets. https://data.worldobesity.org/publications/WOF-Missing-the-2025-GlobalTargets-Report-FINAL-WEB.pdf 